\title{
Effects of Partial and Total Sleep Deprivation On Driving Performance
}

Publication No.: FHWA-RD-94-046

February 1999 
The original format of this document was an active HTML page(s). The Federal Highway Administration converted the HTML page(s) into an Adobe ${ }^{\circledR}$ Acrobat $₫$ PDF file to preserve and support reuse of the information it contained.

The intellectual content of this PDF is an authentic capture of the original HTML file. Hyperlinks and other functions of the HTML webpage may have been lost, and this version of the content may not fully work with screen reading software.

\section{Table of Contents}

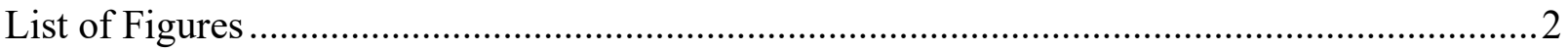

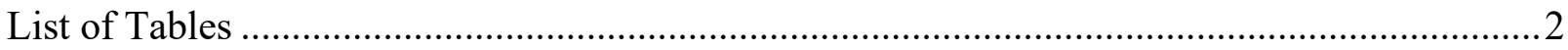

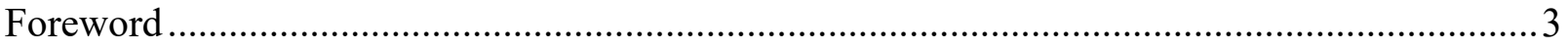

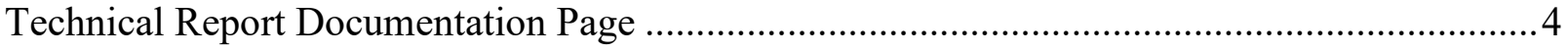

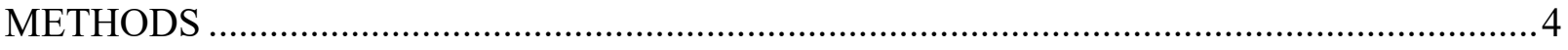

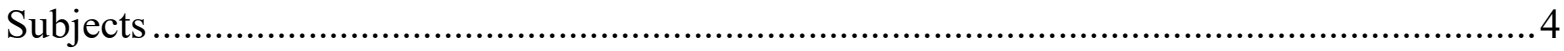

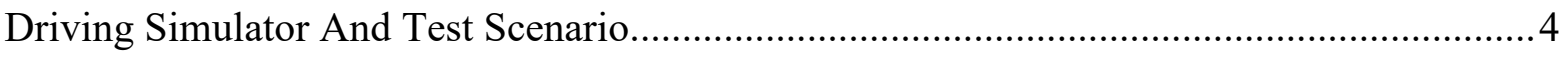

RESULTS \& DISCUSSION ......................................................................................

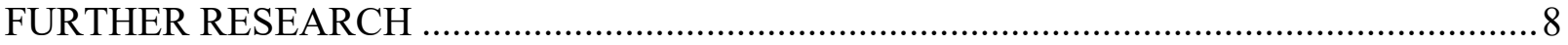

\section{List of Figures}

Figure. The HYSIM control room. ………….................................................................

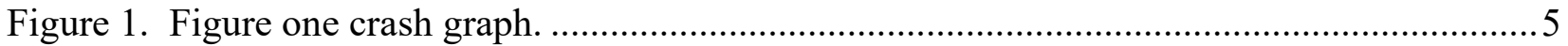

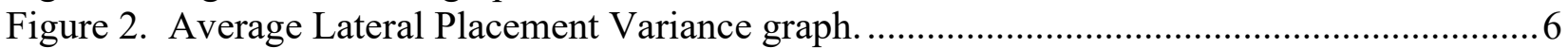

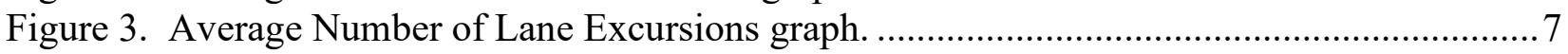

Figure 4. Average Speed by Experimental Day .....................................................................

\section{List of Tables}

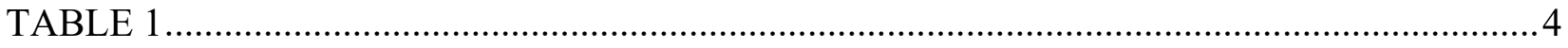




\author{
Robert D. Peters and Esther Kloeppel \\ Science Applications International Corporation (SAIC) \\ Elizabeth Alicandri \\ Federal Highway Administration (FHWA) \\ Jean E. Fox \\ George Mason University
}

Maria L. Thomas, David R. Thorne, Helen C. Sing, and Sharon M. Balwinski

Walter Reed Army Institute of Research

During the period 1989 - 1993, there were an estimated 56,000 crashes annually on U.S. highways in which driver drowsiness/fatigue was cited on the Police Accident Report (PAR) (Knipling and Wang, 1994). During the same five-year period, drowsiness/fatigue was cited as a factor in an annual average of 1357 fatal crashes, resulting in 1544 fatalities (3.6 percent of all fatal crashes annually). These statistics for driver sleepiness and accidents are considered conservative because of differences in state reporting practices, lack of firm evidence as to crash cause, failure of drivers involved in crashes to report that sleepiness played a role in an accident, and lack of citing a significant number of crashes involving a drift out of lane vehicle path as drowsiness-related on the PARs. Driver drowsiness/fatigue has also been cited as a problem for military drivers. Approximately nine percent of wheeled vehicle driving accidents resulting in injury or death during Operations Desert Shield and Desert Storm was attributed to driver drowsiness/fatigue (United States Army Safety Center, 1994). Accidents due to driver sleepiness, caused by sleep deprivation or drug effects, are often assumed to be the result of the driver falling asleep behind the wheel.

Reducing the extent of the drowsy driver problem is critical to improving the safety of our nation's highways. In order to address this issue, the research community must identify driving performance measures that are sensitive to sleep deprivation. These findings will be used as the basis for the development of a method of predicting when sleepiness may put a driver at risk for crashes and countermeasures to alleviate the drowsy driver problem can then be generated and tested.

A study of the Effects of Partial and Total Sleep Deprivation on Driving Performance was conducted jointly by the Federal Highway Administration's (FHWA) Human Factors Laboratory and the Walter Reed Army Institute of Research's (WRAIR). It examined the effects of progressive sleep deprivation on simulated driving performance in the laboratory to assess the rate of accidents and changes in driving performance resulting from sleepiness. Continuous EEG monitoring, video taping, and questionnaire data were recorded to determine if crashes and/or driving errors resulted from falling asleep or some other factor. The primary purpose of the study was to examine the effects of reduced sleep and progressive sleep deprivation on driver accident rates under controlled conditions. Since it would be unsafe to study this under real road conditions, the high-fidelity driving simulator (HYSIM) at TFHRC was used. 


\section{Technical Report Documentation Page}

\section{METHODS}

\section{Subjects}

The twelve subjects, six men and six women between the ages of 26 and 35 , with normal or corrected-tonormal vision, were licensed, non-professional drivers who reported driving a minimum of 8,000 miles per year. Subjects passed a physical examination, drug test, mental states exam and cognitive test, and a test for motion sickness. In addition, subjects maintained a prescribed sleep schedule (at least 8 hours per night) for the seven days prior to the start of the study that was verified by a wrist-worn movement activity monitor. Subjects were non-smokers, and low caffeine users, and did not use stimulants, including caffeine, throughout the study as determined by daily drug screens. Women subjects were not pregnant during the study. Subjects remained in a residential suite with testing chambers at WRAIR during the eight-day study runs and made a total of five trips to FHWA TFHRC for training and tests on the HYSIM. See Table 1 for a description of daily test conditions:

\section{TABLE 1}

\begin{tabular}{|c|c|c|}
\hline $\begin{array}{c}\text { Test } \\
\text { Day }\end{array}$ & $\begin{array}{c}\text { Hours of } \\
\text { Continuous } \\
\text { Wakefulness }\end{array}$ & $\begin{array}{c}\text { Level of } \\
\text { Deprivation }\end{array}$ \\
\hline $\begin{array}{c}\text { Day } \\
\text { One }\end{array}$ & 9 Hours & None \\
\hline $\begin{array}{c}\text { Day } \\
\text { Two }\end{array}$ & 12 Hours & $\begin{array}{c}4 \text { Hours } \\
\text { Sleep }\end{array}$ \\
\hline $\begin{array}{c}\text { Day } \\
\text { Three }\end{array}$ & 36 Hours & $\begin{array}{c}\text { No Sleep for } \\
\text { One Day }\end{array}$ \\
\hline $\begin{array}{c}\text { Day } \\
\text { Four }\end{array}$ & 60 Hours & $\begin{array}{c}\text { No Sleep for } \\
\text { Two Days }\end{array}$ \\
\hline
\end{tabular}

\section{Driving Simulator And Test Scenario}

Testing was conducted in the HYSIM at TFHRC in McLean, VA.

The HYSIM is a high-fidelity, fully-interactive, fixed-base driving simulator. It is organized around a full sized car cab, with a computer-generated image projected onto a 70 by 35 degree front screen. The test scenario consisted of a 20-mile rural loop divided into eight octants with four different start points. On each day, subjects entered at a different start point. Start points were counterbalanced between subjects and across the four days for each subject. The octants differed in the number of lanes and the speed limit; using all combinations of speed limit ( $35 \mathrm{mph}$ vs. $55 \mathrm{mph}$ ) and number of lanes (2 lanes vs. 4 lanes) twice. 


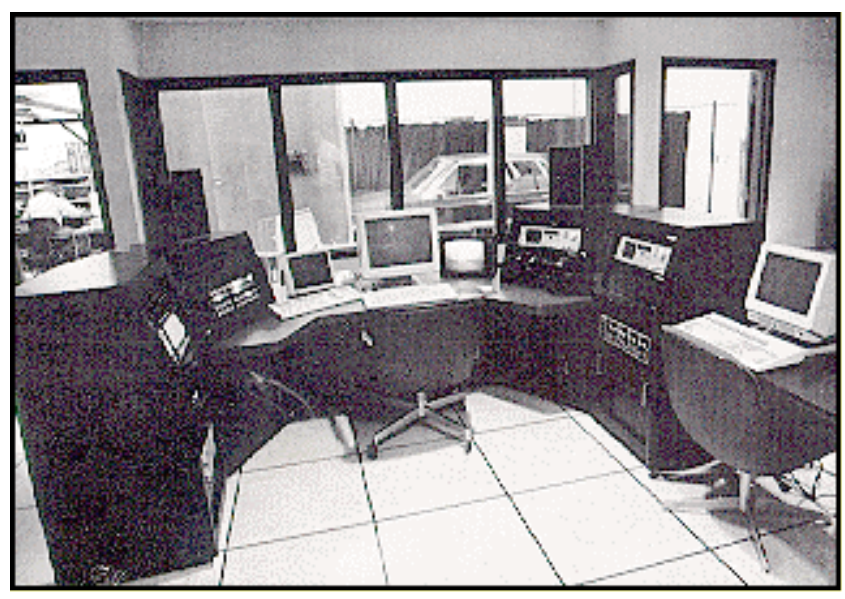

\section{Procedure}

Subjects spent the week at WRAIR. They were driven to TFHRC every afternoon to drive the HYSIM. Subjects drove the simulator between 2:00 and 4:00pm, the afternoon circadian trough. Polysomnographic monitoring electrodes were applied to all the subjects at the beginning of the study and remained on continuously for the entirety.

\section{Figure. The HYSIM control room.}

\section{RESULTS \& DISCUSSION}

The driving data provide confirmation that sleepiness is a significant factor leading to off-road accidents. Accident rates showed a small increase after a moderate reduction in the previous night's sleep (4h vs. $8 \mathrm{~h})$, and a marked increase with progressive sleep deprivation.

See Figure 1.

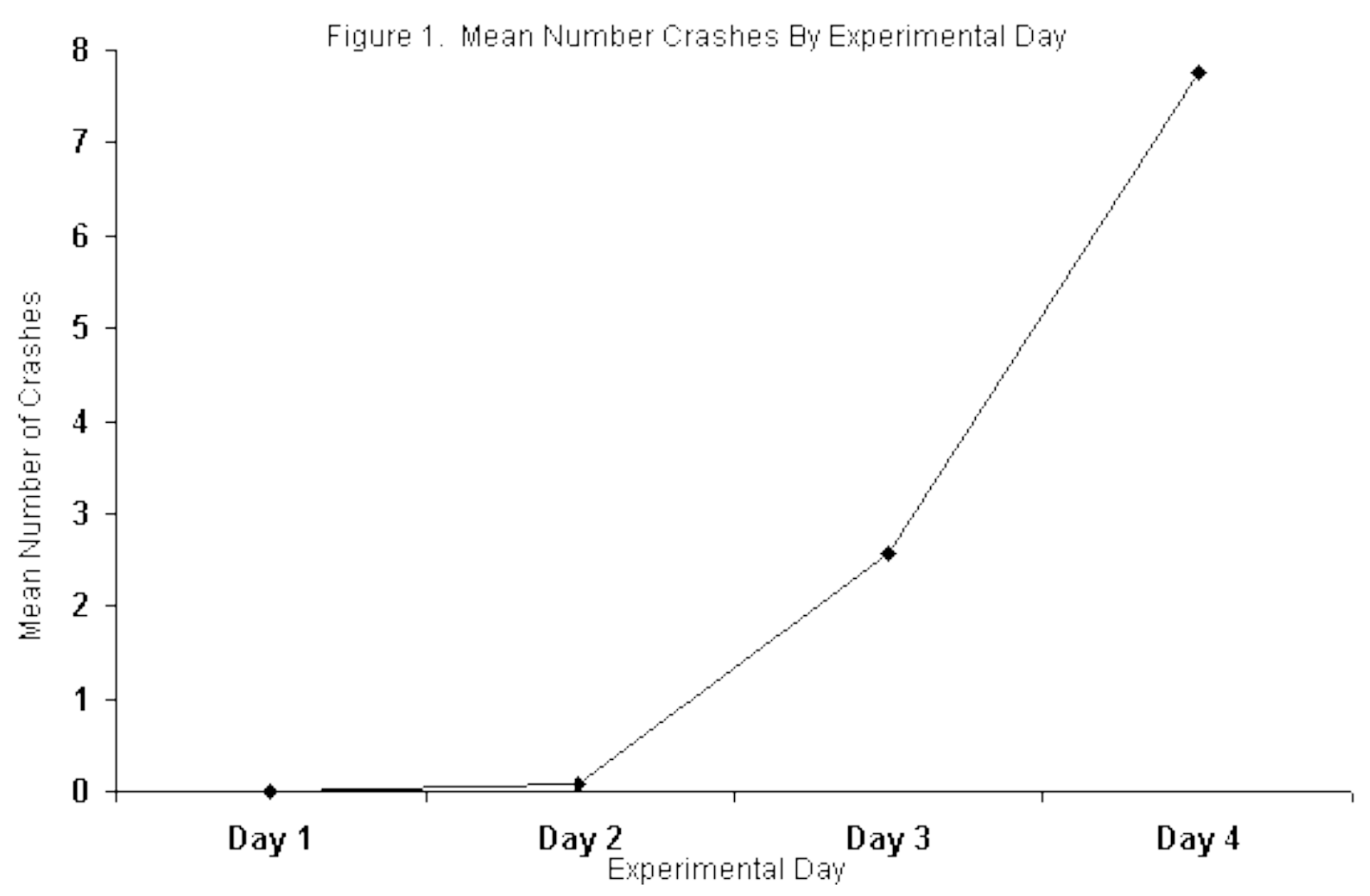

\section{Figure 1. Figure one crash graph.}

While the increase in accident rate after partial sleep deprivation did not reach statistical significance, it should be noted that a relatively small sample of subjects was used and that continuous driving for long periods of time was not assessed. A related issue remains to be investigated on the effects of chronic 
partial sleep deprivation (several nights of restricted sleep resulting in a "sleep debt") on driving performance, which we hypothesize may result in off-road driving accidents comparable to total sleep deprivation.

Other driving performance variables significantly affected by progressive sleep deprivation were:

1. lateral placement variance

2. lane excursions

3. speed

See Figures 2-4.

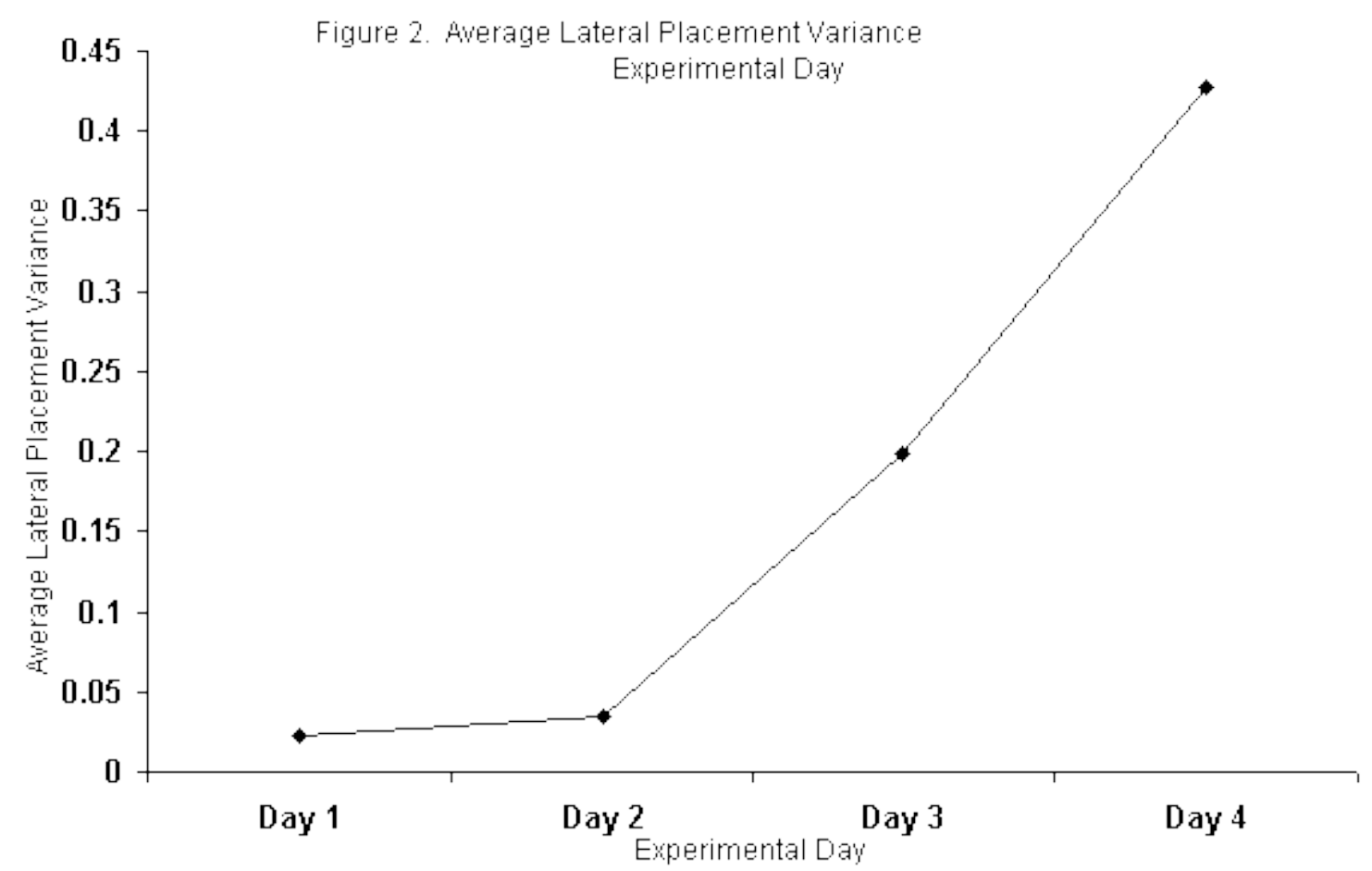

Figure 2. Average Lateral Placement Variance graph. 


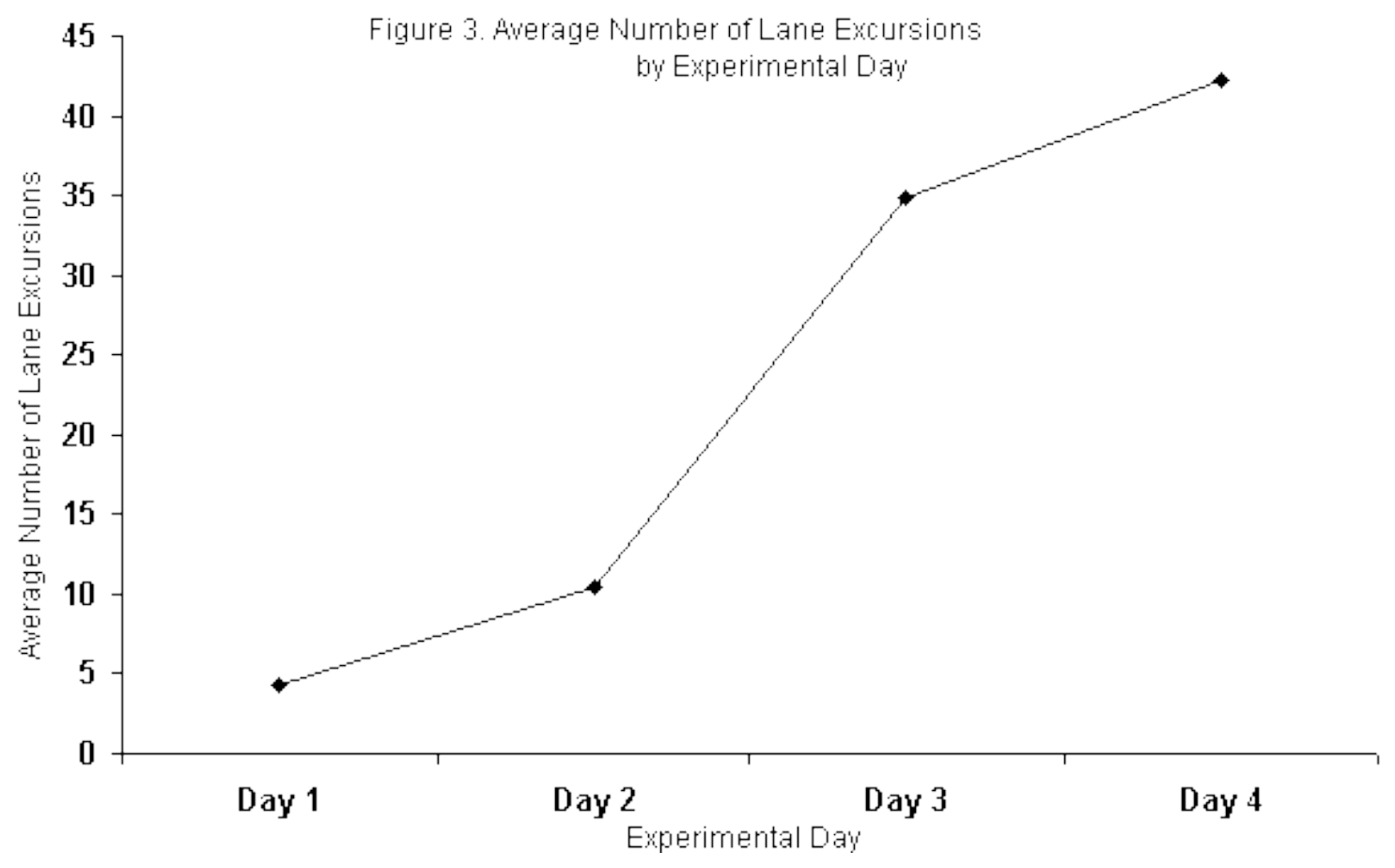

Figure 3. Average Number of Lane Excursions graph.

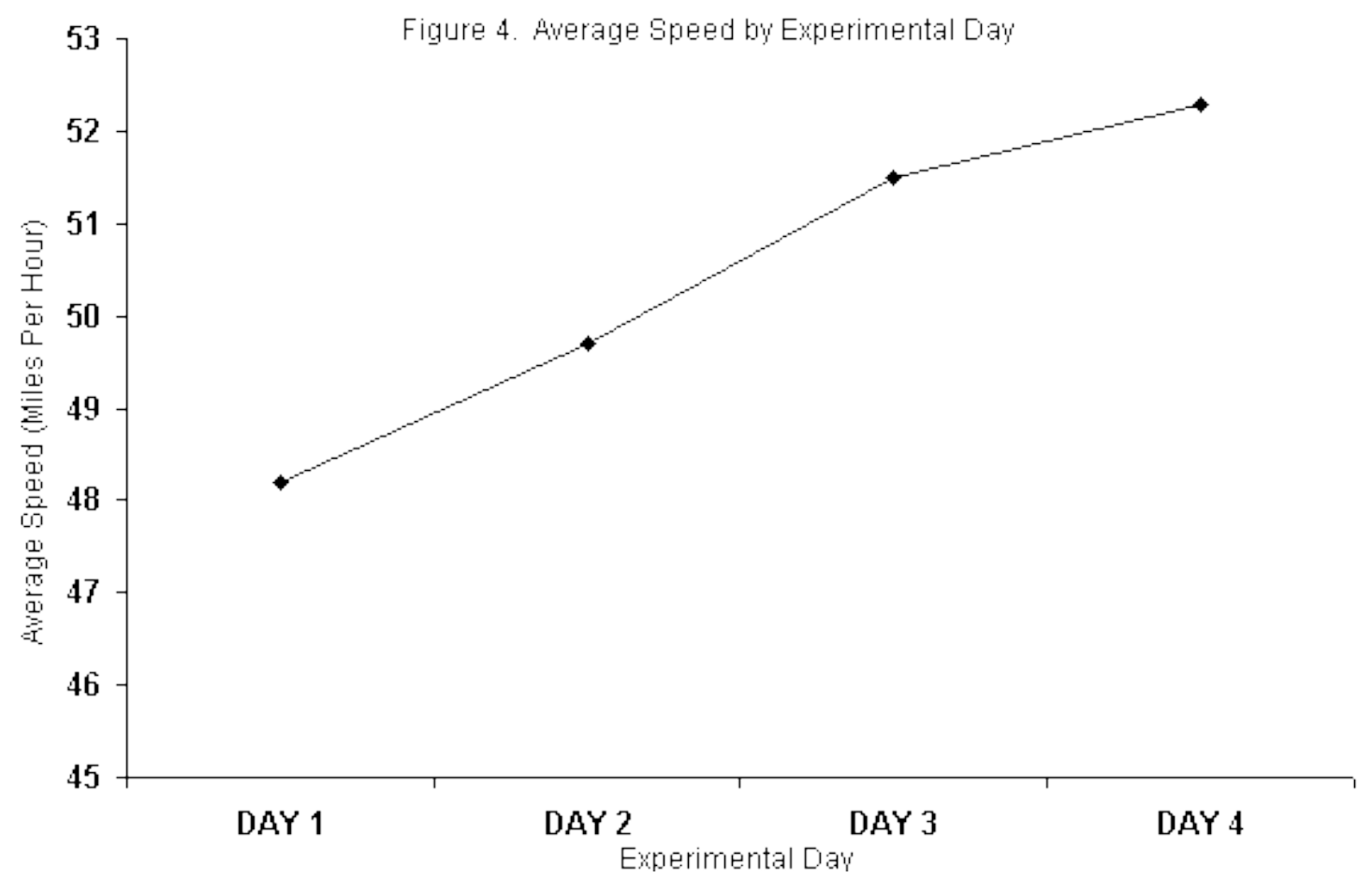

Figure 4. Average Speed by Experimental Day.

In addition, both lateral placement variance and lane excursions were highly predictive of impending crashes. 
The primary objectives of this effort, to identify driving performance measures that are sensitive to sleep deprivation and to predict crashes based on these measures, were met. The effects of significant amounts of sleep deprivation are clearly shown in the driving performance data. Variables clearly related to highway safety (number of crashes, number of lane excursions) were unacceptably high on Days 3 and 4 (36 and 60 hours awake, respectively). More subtle measures of highway safety (speed and lateral placement variance) are also linked to sleep deprivation. Although some trends appeared, none of the variables were significantly effected by partial sleep deprivation. This may be due to the sample used in this study: young, very healthy, non-medicated subjects with no sleep debt when entering the study. Further research on the effects of sleep deprivation should include a wider age range of subjects, subjects with some sleep debt, and testing during the primary circadian trough (4 -6 AM).

Although quantitative extrapolations cannot be made from the simulator data. The simulator allowed subjects to resume driving immediately after an off-road accident or collision with another vehicle. Thus the absolute number of recorded accidents far exceed what could occur in an actual 40-minute drive. Although this aspect of simulation may be viewed as "unrealistic", it increases measurement sensitivity and provides the ability to detect low-probability events. The findings obtained here lend support to the idea of rumble strips or other devices to warn, alert, or awaken drivers as they approach the road edge. In the present study, when a subject had an off-road accident, a loud crash noise coincided with the event. This noise was sufficient to alert or awaken the subject, after which the subject would continue to drive. Yet, with total sleep deprivation, even after being alerted/awakened after the first off-road accident, several subjects repeatedly incurred additional off-road accidents. This finding suggests that highway design aids that use noise or motion to alert/awaken drivers (such as rumble strips), while sufficient to instantaneously alert or awaken the driver, might not be sufficient to maintain continuing alertness in those with further distance to drive.

Preliminary countermeasures for drowsy driving problems can be defined from this effort. Clearly, after a crash occurs, it is too late for interventions to warn drivers of their dangerously drowsy states. Lane excursions have such significant safety implications that they are also too late to be of use. Lateral placement changes, however, can be calculated as the driver operates the vehicle, and a warning could be considered when this variable exceeds a certain threshold. Given the high predictive utility of lateral placement variance, this driving performance measure shows significant promise as a method for early detection of sleepiness that may lead to crashes.

FURTHER RESEARCH

As a result of sleep deprivation studies, methods of predicting critical performance failures due to sleepiness that may lead to accidents are being developed. Knowledge of the variables leading up to an accident brings us one step closer to the development of highway engineering and in-vehicle drowsy driver warning systems and devices to prevent the drowsy driver from endangering themselves and others. The next logical step is to conduct a neural net analysis of the data. The neural net would be used as a pattern recognizer and both driving performance data that precipitated crashes and performance data that did not lead to crashes would be fed into the neural net. The neural net would be able to differentiate between patterns of driving performance data and recognize patterns in the driving performance data that had a high likelihood of resulting in a crash. This demonstration, if successful, would lend strong support for the development of a neural net in-vehicle-based system for detecting and warning drowsy drivers of potential danger and hopefully prevent fatigue-related accidents. 